\title{
Differential Evolution Immunized Ant Colony Optimization Technique in Solving Economic Load Dispatch Problem
}

\author{
N. A. Rahmat, I. Musirin \\ Universiti Teknologi MARA, Malaysia \\ Email: azamrahmat@gmail.com, i_musirin@yahoo.co.uk
}

Received 2013

\begin{abstract}
Since the introduction of Ant Colony Optimization (ACO) technique in 1992, the algorithm starts to gain popularity due to its attractive features. However, several shortcomings such as slow convergence and stagnation motivate many researchers to stop further implementation of ACO. Therefore, in order to overcome these drawbacks, ACO is proposed to be combined with Differential Evolution (DE) and cloning process. This paper presents Differential Evolution Immunized Ant Colony Optimization (DEIANT) technique in solving economic load dispatch problem. The combination creates a new algorithm that will be termed as Differential Evolution Immunized Ant Colony Optimization (DEIANT). DEIANT was utilized to optimize economic load dispatch problem. A comparison was made between DEIANT and classical ACO to evaluate the performance of the new algorithm. In realizing the effectiveness of the proposed technique, IEEE 57-Bus Reliable Test System (RTS) has been used as the test specimen. Results obtained from the study revealed that the proposed DEIANT has superior computation time.
\end{abstract}

Keywords: Ant Colony Optimization (ACO), Differential Evolution (DE), Differential Evolution Immunized Ant Colony Optimization (DEIANT)

\section{Introduction}

In 1992, Marco Dorigo introduced a probabilistic algorithm known as Ant Colony Optimization (ACO) technique. In his $\mathrm{PhD}$ thesis, he described that ACO resembles the natural behavior of a colony of ant during their random expedition to find the best path between their nest and food source. The ant will deposit a chemical trace known as pheromone. The pheromone will act as the stimulant to attract more ants to utilize on the same path. Any less-traveled paths will be forgotten since their pheromone traces has been evaporated. Marco Dorigo employed this behavior into his research to solve the travelling salesmen problem (TSP) [1]. Since, ACO has attracted many researchers to employ the algorithm into their research. Mohd Rozeli Kalil et. al successfully implements ACO to gain maximum loadability in voltage control study [2]. Ashish Ahuja and Anil Pahwa [3] stated in their research that ACO significantly minimized the loss in a distribution system. Moreover, D. Nualhong et. al utilizes ACO in his research to solve the unit commitment problems [4]. However, further research on the algorithm indicates that ACO suffers from several short- comings. H. B. Duan, et al stated in his research that ACO may experience stagnation due to its positive feedback strategy. The researcher also highlighted that the random selection strategy of ACO makes the algorithm sluggish [5]. Another researcher claimed that ACO has slow convergence rate [6-7].

Another attractive optimization technique is the Differential Evolution (DE). DE was created by Storn and Price in 1995 [8]. As a typical Evolutionary Algorithm (EA), DE was used to solve stochastic, non-differentiable, non-linear, multi-dimensional and population-based optimization problem [9]. The algorithm was also used to solve a Chebychev Polynomial Fitting Problem during the First International Contest on Evolutionary Computer ( $1^{\text {st }}$ ICEO) in Nagoya. As a typical Evolution Algorithm, DE comprises of mutation, crossover, and selection process. In 1997, Storn and Price stated that DE is much better than Simulated Annealing and Genetic Algorithm [10]. As such, DE has become the candidate technique to optimize Neural Network Learning, Radio Network design, multiprocessor synthesis and gas transmission network design. Therefore, this paper presents Differential 
Evolution Immunized Ant Colony Optimization (DEIANT) technique in solving economic load dispatch problem. The aim of the proposed technique is to alleviate the slow convergence, and stagnation experienced in the traditional ACO through the application of DE. The performance of DEIANT will be evaluated by using the algorithm to solve and optimize economic load dispatch problems. Performance evaluation of the proposed DEIANT revealed that the proposed technique is superior than the traditional ACO in terms of achieving optimal solution and computation time.

\section{Differential Evolution Immunized Ant Colony Optimization Formulation}

The capability to achieve fast convergence has been identified as the attractive feature of DE [10]. This feature will be used to compensate the stagnation of ACO algorithm. The modification of ACO algorithm will be focusing on the pheromone updating rule which is subjected to cloning, mutation, crossover, and selection process. Figure 1 depicts the overall structure and processes of Differential Evolution Immunized Ant Colony Optimization (DEIANT) algorithm. DEIANT is configured from the combination of original ACO, combined with DE and cloning process. ACO search agent will initialize random tours and deposit pheromone layers. The algorithm will update the pheromone level at each iteration. Cloning process will increase the pheromone amount by generating the exact copies of the pheromone layer. To enhance the pheromone layer, DE will mutate and crossover the pheromone layer, thus increasing the diversity of the pheromone. ACO will evaluate the fitness of the pheromone layer.

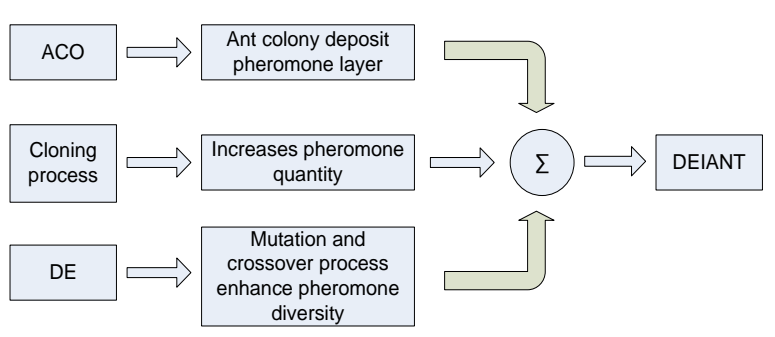

Figure 1. Structural diagram of DEIANT

\subsection{Initialization}

DEIANT consist of several classical ACO parameters. Therefore, similar to ACO technique, the initial number of ant, nodes, pheromone decay parameter, $\alpha$, and the initial pheromone level, $\tau_{0}$ will be heuristically initialized. The maximum distance travelled by each ant, $d_{\text {max }}$, is subjected to a constraint in order to avoid large computation time. $d_{\max }$ is obtained by calculating the longest distance travelled by the ants. Each ant will tour and select the next unvisited node until all nodes have been visited.

\subsection{Apply State Transition Rule}

The next unvisited node is chosen according to the state transition rule, s. Each node can only be visited once. The ant that was positioned at node(r) will go to the next node(s).

$$
P_{k}(r, s)=\left\{\frac{[\tau(r, s)] \cdot\left[\eta(r . s)^{\beta}\right]}{\sum \mu J_{k(r)}[\tau(r, u)] \cdot\left[\eta(r . u)^{\beta}\right]}\right.
$$

\subsection{Apply Local Updating Rule}

Altering the level of pheromone deposition is done during the construction of solution. The amount of pheromone is either increased or decreased to vary the attractiveness of the route via the evaporation rate, $\rho$. Dorigo stated that parameter $\rho$ is needed to avoid the algorithm converge pre-maturely. The parameter $\rho$ act as a multiplier and is set between 0 to 1 . In this research, the evaporation rate is set to 0.7 , meaning that at every iteration; the pheromone will be reduced by 0.7 .

\subsection{Pheromone Cloning}

The cloning process from Artificial Immune System is implemented into DEIANT. The pheromone level will be duplicated to increase pheromone population and diversity. Figure 2 below depicts the cloning of the original pheromone level.

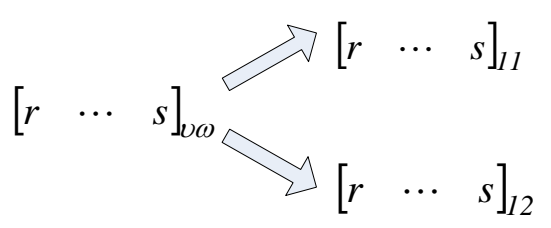

Figure 2. Pheromone cloning process

Where $v$ represents the set of pheromone that will be cloned, and $\omega$ represents the cloned pheromone set. Both $v$ and $\omega$ can be written as $v=1, \ldots, V$ and $\omega=1, \ldots W$, respectively.

\subsection{Pheromone Mutation}

The pheromone mutation process is used to enhance the pheromone layer over the visited node during ant exploration. The Gaussian Distribution Equation was utilized to mutate the pheromone level as in (2): 


$$
X_{i+m, j}=X_{i, j}+N\left(0, \beta \cdot\left(X_{j \max }-X_{j \min }\right) \cdot \frac{f_{i}}{f_{\text {max }}}\right)
$$

Where

$X_{i+m, j}$ : Pheromone mutation function

$X_{j \min }$ : Smallest visited node

$X_{j \text { max }}$ : Largest visited node

$f_{i} \quad$ : Distance travelled by ant

$f_{\max }$ : Longest distance travelled by ant

\subsection{Crossover}

Crossover operation is implemented to emphasize the diversification vector of the pheromone trail. The mutated pheromone trail and the original one will merge together by applying a binomial distribution known as the crossover operation. In this algorithm, the original and mutated pheromone level will be resorted within the same matrix. The crossover matrix, $X_{g i}$ is sorted in descending order, as shown below:

$$
X_{g i}=\left[\begin{array}{ccc}
n & \cdots & n-1 \\
\vdots & \ddots & \vdots \\
n-1 & \cdots & n-m
\end{array}\right]
$$

Where $n$ is the highest pheromone level for the tour, and $n-m$ is the lowest pheromone level.

\subsection{Selection}

Trial pheromone trail, $\rho_{t}$ is the product of the crossover process. The algorithm will now select the required trail according to this rule:

$$
\rho_{t}=\left\{\begin{array}{c}
\rho_{t}, \text { if pheromone level, } \rho \leq \alpha \\
\rho, \text { otherwise }
\end{array}\right.
$$

Where $\alpha$ is the pheromone decay parameter.

Basically, the selection process will specify the acceptable condition to accept the pheromone level. The trail with a higher pheromone level will be selected. The trail with fewer pheromone levels will be discarded.

\subsection{Control Variable Calculation}

The control variable $x$, is computed by applying the following equation:

$$
x=\frac{d}{d_{\max }} \cdot x_{\max }
$$

Where:

$d \quad$ : distance for every ants tour

$d_{\max }$ : maximum distance for every ants tour

$x_{\max }$ : maximum of $x$

Variable $x$ will become the multiplier to calculate the objective function. In this research, variable $x$ is multiplied with the cost function (7).

\subsection{Global Updating Rule}

After all ants have finished performing their random exploration, the best ant of the colony will be selected. The best ant is carrying the data of the best tour, and thus, the data will be stored. The following equation is applied to update the pheromone level globally:

$$
\tau(r, s) \leftarrow(1-\alpha) \tau(r, s)+\alpha . \Delta \tau(r, s)
$$

The globally best tour will be selected as the first node of the next iteration.

\subsection{End Condition}

The DEACO algorithm will halt the iteration when the maximum number of iteration $\left(t_{\max }\right)$ has been reached and all ants have completed their tours. If a better path is discovered, it will be used as the reference. Only one ant will find the optimal path.

\section{Economic Load Dispatch Formulation}

Economic load dispatch is the operation of determining the optimal output that must be produced by the generation facilities to feasibly satisfy the load demand [12]. Therefore, the key objective of economic load dispatch is to reduce the operating cost of each generating unit in the power system. The operation cost can be calculated by utilizing equation (7):

$$
\text { cost }=\sum_{i}^{N g} F_{i}\left(P_{i}\right)
$$

Where $N g$ is the number of generating unit. $F_{i}\left(P_{i}\right)$ is the cost function, and $P_{i}$ is the real power output of the unit $I$, measured in MW. $F_{i}\left(P_{i}\right)$ is usually approximated by a quadratic function:

$$
F_{i}\left(P_{i}\right)=a_{i} P_{i}^{2}+b_{i} P_{i}+c
$$


Where $a_{i}, b_{i}$, and $c_{i}$ are the cost coefficients of generator $i$. The above equation is subjected to both the equality and inequality constraints.

The system power loss can be calculated by using the power flow equation below (19):

$$
P_{L}=\sum_{i}^{N} \sum_{j}^{N} P_{i} B_{i j} P_{j}+\sum_{i}^{N} B_{o i} P_{i}+B_{o o}
$$

Where $B_{i j}, B_{o i}$, and $B_{o o}$ are the $B$-loss coefficient. The cost function is given by equation (26):

$$
C_{n}=a+b P_{n}+c P_{n}^{2}
$$

The followings are the generators' operating costs for IEEE 57-Bus System. These equations are derived from equation (10):

$$
\begin{gathered}
C_{1}=400+7.0 P_{1}+0.0070 P_{1}^{2} \\
C_{2}=200+10.0 P_{2}+0.0095 P_{2}^{2} \\
C_{3}=220+8.5 P_{3}+0.0090 P_{3}^{2} \\
C_{6}=200+11.0 P_{6}+0.0090 P_{6}^{2} \\
C_{8}=240+10.5 P_{8}+0.0080 P_{8}^{2} \\
C_{9}=200+12.0 P_{9}+0.0075 P_{9}^{2} \\
C_{12}=180+10.0 P_{12}+0.0068 P_{12}^{2}
\end{gathered}
$$

Where $C_{1}, C_{2}, C_{3}, C_{6}, C_{8}, C_{9}$ and $C_{12}$ are the operating cost functions for generator 1 , generator 2 , generator 3 , generator 6 , generator 8 , generator 9 , and generator 12 respectively.

The total operating cost, $C_{T}$ can be formulated as:

$$
C_{T}=C_{1}+C_{2}+C_{3}+C_{6}+C_{8}+C_{9}+C_{12}
$$

The total operating cost is the objective function for this research. The objective is to cut-down the total operating cost, while preserving the system constraints within the permissible limits. While minimizing the operating cost, it was initially estimated that the power loss will be reduced as well. Power loss is the marginal difference between the power produced, and the power sold to the consumer [13].

\section{Results and Discussion}

The DEIANT algorithm was developed by using MATLAB. The algorithm was used to optimize economic load dispatch problem. The research was conducted on IEEE-57 bus system. The system contains seven generating units. The load was varied to assess the performance of the proposed algorithm. The objectives are to minimize the total generating cost, to reduce transmission loss, and to reduce the computation time. Comparisons were made between DEIANT and the original ACO. Table 1 tabulates the simulation results of classical ACO algorithm. Table 2 tabulates the simulation results of the newly developed DEIANT algorithm. Note that the load, designated by $Q_{d}$ at Bus 10 was varied between 0 to 25MVAR to see the performance of DEIANT in solving economic load dispatch problem. The comparison of DEIANT and classical ACO undoubtedly points out that the new algorithm successfully

\begin{tabular}{|c|c|c|c|c|c|c|c|c|c|c|}
\hline $\mathbf{Q}_{d}$ (MVAR) & $\mathbf{P}_{1}(\mathbf{M W})$ & $\mathbf{P}_{2}(\mathbf{M W})$ & $\mathbf{P}_{3}(\mathbf{M W})$ & $\mathbf{P}_{6}(\mathbf{M W})$ & $\mathbf{P}_{8}(\mathbf{M W})$ & $\mathbf{P}_{9}(\mathbf{M W})$ & $\mathbf{P}_{12}(\mathrm{MW})$ & $\mathbf{P}_{\text {loss }}(\mathbf{M W})$ & $\begin{array}{l}\text { Total Cost } \\
(\$ / \mathbf{h})\end{array}$ & Time (s) \\
\hline 0 & 137.1095 & 89.8488 & 45.1114 & 75.8669 & 463.3333 & 100 & 358.7308 & 19.2007 & 41842 & 30.63371 \\
\hline 5 & 138.5126 & 96.3783 & 45.3754 & 74.8029 & 458.1759 & 96.2979 & 360.593 & 19.336 & 41856 & 70.29794 \\
\hline 10 & 138.4993 & 96.3443 & 45.3752 & 74.7286 & 458.1097 & 96.6145 & 360.495 & 19.3666 & 41858 & 52.4811 \\
\hline 15 & 138.4077 & 96.3373 & 45.377 & 74.6806 & 458.0548 & 96.9501 & 360.4142 & 19.4217 & 41860 & 67.40478 \\
\hline 20 & 138.4437 & 96.2948 & 45.3759 & 74.5882 & 457.9824 & 97.268 & 360.3066 & 19.4597 & 41862 & 34.64456 \\
\hline 25 & 138.4127 & 96.2995 & 45.366 & 74.7954 & 457.8748 & 97.4865 & 360.121 & 19.5559 & 41866 & 53.20833 \\
\hline
\end{tabular}
outperforms the classical ACO. Varying the load gives less impact to the performance of DEIANT. For example, when $Q_{d}$ is set to 20MVAR, DEIANT generates the total operating cost of $41855 \$ / \mathrm{h}$. The cost is economical than the one calculated by classical ACO (41862 $\$ / \mathrm{h})$, giving a price drop of $0.0167 \%$. If the load was set to 15MVAR, the idea is the same; DEIANT only generates lower power loss.

Table 1. Simulation results of classical ACO

Table 2. Simulation results of DEIANT 


\begin{tabular}{|c|c|c|c|c|c|c|c|c|c|c|}
\hline $\begin{array}{c}\mathbf{Q}_{\mathrm{d}} \\
\text { (MVAR) }\end{array}$ & $\mathbf{P}_{1}(\mathbf{M W})$ & $\mathbf{P}_{2}(\mathbf{M W})$ & $\mathbf{P}_{3}(\mathbf{M W})$ & $\mathbf{P}_{6}(\mathbf{M W})$ & $\mathbf{P}_{8}(\mathbf{M W})$ & $\mathbf{P}_{9}(\mathrm{MW})$ & $\mathbf{P}_{12}(\mathbf{M W})$ & $\mathbf{P}_{\text {loss }}(\mathrm{MW})$ & $\begin{array}{c}\text { Total Cost } \\
(\$ / \mathbf{h})\end{array}$ & Time (s) \\
\hline 0 & 137.3725 & 90.9657 & 45.045 & 74.2499 & 463.3627 & 100 & 358.7523 & 18.9481 & 41832 & 4.317901 \\
\hline 5 & 137.349 & 91.0764 & 45.0431 & 74.2626 & 463.3531 & 100 & 358.6874 & 18.9716 & 41832 & 4.539834 \\
\hline 10 & 138.7015 & 97.2722 & 45.324 & 73.6004 & 458.3919 & 96.009 & 360.6952 & 19.1941 & 41851 & 4.854118 \\
\hline 15 & 138.6771 & 97.2475 & 45.3171 & 73.5275 & 458.3209 & 96.3482 & 360.5982 & 19.2362 & 41853 & 4.778885 \\
\hline 20 & 138.6519 & 97.2263 & 45.31 & 73.4555 & 458.25 & 96.6919 & 360.5016 & 19.2872 & 41855 & 5.184511 \\
\hline 25 & 138.626 & 97.2089 & 45.3028 & 73.3846 & 458.1796 & 97.04 & 360.4055 & 19.3474 & 41857 & 5.328133 \\
\hline
\end{tabular}

Moreover, at 20MVAR load adjustment, the classical ACO computed power loss of 19.4597MW. However, DEIANT effectively reduces it to $19.2872 \mathrm{MW}$, with a significant difference of $172.5 \mathrm{~kW}$. Furthermore, DEIANT possesses faster computation time than the classical ACO. For instance, classical ACO requires about 35 seconds to optimize the objective function, but DEIANT optimize the objective function in just five seconds. This implies that DEIANT can achieve optimal solution at a faster rate, although the algorithm is more complex and sophisticated than the classical ACO.

\section{Conclusion}

This study demonstrates the development of a new algorithm termed as Differential Evolution Immunized Ant Colony Optimization (DEIANT). Through the combination of DE, ACO and cloning process, this new algorithm has successfully overcome the drawbacks of classical ACO algorithm. The good performance of DEIANT was verified by optimizing economic load dispatch problem. Comparisons with classical ACO imply that DEIANT effectively cuts-down the operating cost while reducing the power loss. The optimization process was performed with a faster speed than the classical ACO. Future development will be focusing on the modification to increase the convergence speed of the algorithm.

\section{Acknowledgement}

The authors would like to acknowledge The Research Management Institute (RMI) UiTM, Shah Alam and Ministry of Higher Education Malaysia (MOHE) for the financial support of this research. This research is supported MOHE under the Fundamental Research
Grant Scheme (FRGS) with project code: 600-RMI/ST/FRGS 5/3/Fst (163/2010).

\section{REFERENCES}

[1] Ying-Tung Hsiao, Cheng-LongChuang and Cheng- Chih Chien, "Ant Colony Optimization for Best Path Planning," International Symposium on Communications and Information Technologies 2004 (ISCIT 2004), Sapporo, Japan, 26-29 October 2004, pp. 109-113.

[2] Mohd Rozely Kalil, Ismail Musirin, Muhammad Murtadha Othman, "Maximum Loadability in Voltage Control Study Using Ant Colony Optimization Technique", IEEE First International Power and Energy Conference (PECon2007), 28-29 Nov. 2006, pp. 240-245.

[3] Ashish Ahuja and Anil Pahwa, "Using Ant Colony Optimization for Loss Minimization in Distribution Networks”, 37th Annual North American Power Symposium, 2005, 23-25 Oct. 2005, pp. 470- 474.

[4] D. Nualhong, et al., "Diversity Control Approach to Ant Colony Optimization for Unit Commitment Problem," in TENCON 2004. 2004 IEEE Region 10 Conference, 2004, pp. 488-491 Vol. 3.

[5] H.B. Duan and D.B. Wang, "a novel improved ant colony algorithm with fast global optimization and its simulation," Information and Control, vol.33, pp. 241-244, April 2004.

[6] Linda Slimani and Tarek Bouktir, "Economic Power Dispatch of Power System with Pollution Control using Multiobjective Ant Colony Optimization”, International Journal of Computational Intelligence Research (IJCIR) 2007, Vol. 3, No. 2, pp. 145-153.

[7] R. Bhavani, G. Sudha Sadasivam, and R. Kumaran, "A novel parallel hybrid K-means-DE-ACO clustering approach for genomic clustering using MapReduce," in Information and Communication Technologies (WICT), 2011 World Congress on, 2011, pp. 132-137.

[8] R. Storn and K. Price, "Differential Evolution - A Simple and Efficient Adaptive Scheme for Global Optimization Over Continuous Spaces”, Technical Report TR-95-012, ICSI, March 1995.

[9] K.P. Wong and Z.Y. Dong, "Differential Evolution, an AlternativeApproach to Evolutionary Algorithm”, in K.Y. Lee edt. 
Intelligent Optimization and Control for Power Systems, IEEE Publishing, invited chapter, Nov. 2005.

[10] Storn R., Price K.: 'Differential Evolution - A Simple and Efficient Adaptive Scheme For Global Optimization Over Continuous Space”, Journal of Global Optimization, 1997.

[11] N. A. Rahmat, I. Musirin (2012). Differential Evolution Ant Colony Optimization Technique (DEACO) In Solving Economic Load Dispatch Problem. IEEE Internation Power Engineering and Optimization.
[12] S. M. V. Pandian and K. Thanushkodi, "Solving Economic Load Dispatch Problem Considering Transmission Losses by Hybrid EP-EPSO Algorithm for Solving Both Smooth and Non-Smooth Cost Function," International Journal of Computer and Electrical Engineering, vol. 2, 2010

[13] M. Basu, "Artificial Immune System for Dynamic Economic dispatch," Electrical Power and Energy System, vol. 33, pp. 131-136, 7 June 2010 